\title{
Una Experiencia de Intervención Psicosocial en Cultura Positiva de Seguridad en una Empresa Productiva Chilena
}

\section{AN EXPERIENCE OF PSYCHOSOCIAL INTERVENTION IN POSITIVE SAFETY CULTURE IN A CHILEAN PRODUCTION COMPANY}

\author{
Héctor Lillo Sepúlveda', Andrés Jiménez Figueroa², Maria Doris Méndez Campos², Emilio Moyano-Díaz², Gonzalo Palomo-Vélez². \\ 1. Psicólogo, Gendarmería de Chile, Linares. \\ 2. Psicólogo, Académico e Investigador Grupo de Investigación en Calidad de Vida y Ambientes Saludables, Facultad de Psicología, Universidad de Talca. \\ 3. Psicólogo, Universidad de Talca.
}

\begin{abstract}
RESUMEN
La reducción de la accidentalidad y la búsqueda de conductas seguras en el trabajo suponen logros apreciados por las organizaciones hoy en día. Aquí se reporta una experiencia de intervención en una empresa productiva chilena desde el Modelo de Cultura Positiva hacia la Seguridad (MCPS), el cual entiende la cultura organizacional como compuesta por dos componentes principales: el clima de seguridad y el sistema de gestión de la seguridad y salud laboral. 435 trabajadores que participaron del proceso de intervención de 11 meses fueron evaluados -antes y después de la intervención- en términos de su compromiso organizacional, satisfacción laboral y cultura positiva hacia la Seguridad (228 durante el pre-test y 207 durante el pos-test). Los resultados indican que la intervención tuvo efectos positivos para cada una de las variables aumentando la mayoría de sus puntuaciones en el pos-test. Asimismo, se observa una reducción en la etapa pos-intervención en cuanto a la frecuencia de accidentes registrados mensualmente en la empresa. Se analizan y contrastan estos resultados con la literatura sobre el tema.
\end{abstract}

(Lillo H, Jiménez A, Méndez M, Moyano-Díaz E, Palomo-Vélez G, 2014. Una Experiencia de Intervención Psicosocial en Cultura Positiva de Seguridad en una Empresa Productiva Chilena. Cienc Trab. Sep-Dic; 16 [51]: 192-197).

Palabras Clave: SEGURIDAD DE EQUIPOS, CULTURA ORGANIZACIONAL, PREVENCIÓN DE ACCIDENTES, CONDICIONES DE TRABAJO.

\section{ABSTRACT}

The reduction in the accident and search for safe behavior at work involve achievements appreciated by organizations today. Here an experience of intervention in a Chilean productive enterprise is reported from the Model Positive Safety Culture at (MCPS), which means the organizational culture as composed of two main components: the climate of safety and safety management system and occupational health. 435 workers who participated in the 11 month intervention process were evaluated -before and after the intervention-in terms of their organizational commitment, job satisfaction and positive culture towards Safety (228 during the pre-test and 207 in the post-test). The results indicate that the intervention had positive effects for each of the variables increasing most of their scores on the posttest. Also, a reduction is observed in the post-intervention phase in the frequency of accidents recorded monthly in the company. We analyze and contrast these results with the literature on the subject.

Key words: EQUIPMENT SAFETY, ORGANIZATIONAL CULTURE, ACCIDENT PREVENTION, WORKING CONDITIONS.

\section{INTRODUCCIÓN}

En el mundo cada año se registran 263 millones de accidentes laborales causando -entre otras cosas- elevados niveles de ausentismo. ${ }^{1,2}$ Aun más, se ha reportado que anualmente unos 312.000 de estos terminarían con consecuencias fatales para el

Correspondencia / Correspondence:

Ps. Mg. Maria Doris Méndez

Facultad de Psicología, Universidad de Talca, Campus Lircay s/n, Talca.

Casilla No. 747 Talca/Chile

Tel: (5671)2201664 • Fax: (5671)2201510

e-mail:dmendez@utalca.cl

Recibido: 17 de Septiembre de 2014 / Aceptado: 16 de Noviembre de 2014 trabajador. $^{3}$ En Chile, la cifra de accidentes laborales registrados el 2012 alcanzó los 264.042 casos, mostrando sólo un leve descenso en comparación a la cifra registrada el año anterior que correspondió a 277.513 accidentes. ${ }^{4}$

Actualmente, una de las prioridades laborales en nuestro país ${ }^{5}$ es reducir las tasas de accidentalidad anual y lograr que la gente interiorice las nociones de seguridad y prevención, a fin de que se conviertan en conceptos imperativos en la sociedad y en el trabajo.

En relación a lo anterior, la educación y el proceso de enseñanzaaprendizaje constituyen el mejor medio para que la seguridad sea un verdadero aporte a la formación de una cultura de seguridad integral, en beneficio de las personas y de la competitividad del país. ${ }^{6}$ La seguridad es considerada hoy un eje fundamental para el correcto funcionamiento de toda actividad productiva. Es así que se ha planteado que las organizaciones que se ocupen de manera seria e integral de la misma tendrán más probabilidades de sobrevivir en su entorno y alcanzar eficaz y eficientemente sus objetivos. ${ }^{7,8}$ 
Las teorias sobre las causas de los accidentes laborales han atravesado distintos estadios de desarrollo, caracterizados principalmente por el foco de a qué o a quién se le atribuye la causalidad del accidente, pudiendo ésta relacionarse con errores mecánicos (período técnico), factores humanos (período de error humano) o una combinación de ambos (período socio-técnico). ${ }^{9}$ Hoy, sin embargo, y desde una perspectiva de cultura organizativa, se asume que los trabajadores no realizan sus actividades ni interactúan con la tecnología de forma aislada, sino que existe coordinación entre ambos, formando un equipo con los demás miembros de la organización con una cultura particular, lo que representa una alternativa integradora de la noción de accidentalidad que permite abarcar diferentes aspectos de la complejidad de las organizaciones. De esta manera, el sistema completo de una organización participaría en la génesis de los accidentes, siendo necesario el planteamiento de intervenciones desde un enfoque psicosocial. ${ }^{10}$

Se han desarrollado diversos modelos y enfoques teóricos para intentar explicar la accidentalidad y permitir el planteamiento de intervenciones en seguridad, entre los cuales está el modelo Causal Psicosocial de Meliá ${ }^{11}$ que integra un conjunto de variables psicosociales a los factores de la tarea y el trabajo considerando, además, las condiciones del mismo. Un segundo enfoque derivado de la perspectiva conductista e implementado en Chile por Ugalde ${ }^{12}$, pone énfasis en la promoción de comportamientos seguros en el trabajo mediante la aplicación de principios conductuales tales como el feedback positivo y el reforzamiento de conductas tendientes a prevenir los accidentes en una organización. El énfasis de esta perspectiva está enfocado al proceder de los trabajadores, no considerando -al menos explícitamente-, factores organizacionales y culturales intervinientes.

Desde un tercer y más amplio modelo denominado Modelo de Cultura Positiva hacia la Seguridad (MCPS) ${ }^{13}$, se plantea que los riesgos de sufrir algún tipo de accidente estarán siempre presentes en los lugares de trabajo y que, por tanto, es necesario crear un ambiente en el cual la seguridad y la prevención de riesgos sean asumidos como procesos conscientes permanentes en la labor productiva de los trabajadores, de manera de adoptar diariamente una postura que resguarde la integridad de estos. En el MCPS, la cultura organizacional se compone por dos componentes principales: el clima de seguridad y el sistema de gestión de la seguridad y salud laboral. El primero se vincula a las actitudes y comportamientos de los trabajadores de la organización, y se puede evaluar por medio del compromiso de la dirección con la seguridad y la salud de la empresa, y la implicación y participación de los trabajadores en materia de prevención en la organización. El segundo, por otro lado, refiere a los elementos situacionales tales como políticas, prácticas y procedimientos asociados a la prevención de riesgos laborales. Este componente lo integrarian 6 dimensiones: desarrollo de una política de prevención; fomento de la participación de los trabajadores en temas de seguridad laboral; formación y promoción de competencias de los trabajadores en materia de prevención; comunicación e información de los riesgos del trabajo; planificación - preventiva y de emergencia- de tareas asociadas a la prevención; y, el control o feedback a través del análisis de las condiciones de trabajo y emergencias o sucesos ocurridos en la organización en comparación a organizaciones similares. ${ }^{8}$

En la literatura han sido descritas distintas intervenciones con base en diferentes aproximaciones teóricas, utilizando diversas organiza- ciones, objetivos y metodologías, considerando -por ejemplofactores conductuales ${ }^{12,14}$, de reflexión y socialización ${ }^{15}$, de información, guía y consultoría ${ }^{16,17}$, entre muchas otras. ${ }^{18}$

Komaki, Barwick, y Scott ${ }^{14}$, utilizando un acercamiento conductual, diseñaron e implementaron una intervención para la prevención de accidentes en una compañía de comida. Los autores codificaron una serie de conductas seguras, las cuales fueron evaluadas a través una pauta de observación durante 25 semanas. La intervención consistió en la diseminación de información sobre cuáles y cómo habían de desarrollarse estas conductas seguras, además de frecuentes refuerzos en forma de feedback. Komaki, Barwick, y Scott ${ }^{14}$ reportaron aumento significativo en la seguridad en la empresa; sin embargo, durante la fase de reversión los indicadores de seguridad volvieron a su línea base. Asimismo, Stave, Törner y Eklöf ${ }^{15}$ también obtuvieron resultados positivos en la realización de conductas seguras mediante la implementación un programa de 7 reuniones entre granjeros suecos durante el transcurso de un año, en las cuales se alentaba la discusión y reflexión sobre el manejo de riesgos en el trabajo y la creación de redes de apoyo entre los mismos. Por otro lado, La Montagne et $\mathrm{al}^{17}$, utilizando uno de los programas propuestos por la Administración de Seguridad Laboral y Salud de Estados Unidos (OSHA por sus siglas en inglés), particularmente el programa Wellworks-2 -que implica entrenamiento y educación al trabajador, consultoría con la gerencia y control de aspectos de inseguridad física de las instalaciones- evaluaron 15 empresas de manufactura, dando cuenta de que la aplicación sostenida de esta clase de intervenciones puede resultar en un mejoramiento de los indicadores de seguridad, pero que el estado inicial y las líneas base de las intervenciones sugieren que tales programas no son necesariamente llevados a cabo de manera sistemática por la empresas del rubro.

Entre los enfoques de tipo cultural, el MCPS aborda la accidentalidad y la seguridad laboral ampliamente y se encuentra en una etapa inicial de desarrollo. Así, aún no existe total certeza sobre el impacto de su aplicación en la reducción de accidentes ni sus eventuales efectos sobre otras variables de carácter psicosocial. Por esta razón, es de interés aquí aplicarlo para probar su eficacia en disminuir los accidentes y, a la vez, analizar otros efectos de su aplicación en los trabajadores, particularmente en variables organizacionales de importancia tales como la satisfacción laboral y el compromiso organizacional de estos. La primera -la satisfacción laboral- refiere a la actitud general de un individuo hacia su trabajo ${ }^{19}$ y se ha descrito que no solo se asociaría, sino que es un predictor del cumplimiento de normas de seguridad. ${ }^{20,21}$ Por otro lado, el compromiso organizacional supone el grado en que un trabajador se identifica y siente satisfacción y orgullo de pertenecer a una organización ${ }^{22}$ y se asociaría -entre otras cosas- con reducción de las tasas de ausentismo y aumento de la productividad $^{23,24}$, ambas características muy deseables para las organizaciones de hoy.

\section{MÉTODO}

Se utilizó un diseño pre-experimental (con pre-test y pos-test) de alcance correlacional para evaluar el efecto de una intervención desde el MCPS en variables de accidentalidad laboral, compromiso organizacional y satisfacción laboral percibida.

Participantes: Se conformó una muestra no probabilística de 435 
trabajadores de una empresa productiva y de exportación de carnes de la zona central de Chile. El pre-test realizado en 2008 (228 trabajadores) y el pos-test de 207, una vez terminado un proceso de intervención de 11 meses durante el año 2009. La diferencia de tamaño muestral entre el pre y pos-test se debe a que algunos trabajadores fueron desvinculados de la empresa por diferentes motivos entre ambas mediciones. Del total de los casos, un $92,4 \%$ es hombre, un $6,4 \%$ es mujer y un $1,1 \%$ no respondió la variable.

\section{Instrumentos}

Escala de Cultura Positiva hacia la Seguridad. Se utilizó la escala de Fernández, Montes ${ }^{13}$ compuesta por 42 ítems con formato de respuesta likert donde 1 corresponde a "muy de acuerdo" y 5 "muy en desacuerdo". De acuerdo a los autores, todos los ítems se proponen neutrales para evitar sesgos. El instrumento evalúa variables asociadas a política de prevención, incentivo a los trabajadores, formación sobre riesgos laborales, comunicación en materia de prevención, planificación preventiva, planificación de emergencia, control interno, técnicas de benchmarking, actitudes de la dirección hacia la gestión, comportamiento de la dirección y participación de los trabajadores. Cada una de las dimensiones presenta un coeficiente de Alfa de Cronbach sobre 0,70 mientras que la confiabilidad general del instrumento es de 0,60. En nuestro conocimiento no existen baremos estandarizados para la escala, de modo que se establecieron terciles para categorizar las puntuaciones globales como bajas (de 1 a 2,74), medias (de 2,75 a 3,46 ) y altas (de 3,47 a 4,90 ).

Cuestionario de Compromiso Organizacional. Cuestionario de Allen y Meyer ${ }^{25}$ para medir compromiso organizacional, el cual se compone de 18 items distribuidos en 3 dimensiones -compromiso afectivo, continuo y normativo- con formato de respuesta tipo Likert, donde 1 corresponde a "totalmente en desacuerdo" y 6 "totalmente de acuerdo", de modo que mayores puntajes en el instrumento denotan mayor compromiso organizacional. Los valores de compromiso organizacional pueden ser categorizados en bajos (de 1,0 a 2,4), medios (de 2,5 a 5,4) y altos (de 5,5 a 7,0). La confiabilidad Alfa de Cronbach para este estudio fue adecuada (de 0,80).

Cuestionario de Satisfacción Laboral S10/12. Cuestionario de Meliá y Peiró ${ }^{26}$, que evalúa satisfacción laboral, se compone de 12 items con formato de respuesta tipo likert donde 1 corresponde a "muy insatisfecho" y 7 "muy satisfecho". El instrumento presenta una estructura de 3 dimensiones:satisfacción con las prestaciones recibidas, satisfacción con el ambiente físico de la empresa, y satisfacción con la supervisión. Los puntajes por dimensión en forma de medias pueden ser categorizados en satisfacción muy baja (de 1 a 2,20), baja (de 2,21 a 3,40), moderada (de 3,41 a 4,60), alta (de 4,61 a 5,80 ) y muy alta (de 5,81 a 7,0). La confiabilidad del cuestionario es adecuada, presentando un Alfa de Cronbach de 0.88 .

Accidentalidad Laboral. Medida a partir de la frecuencia mensual de accidentes laborales reportados antes, durante y después de la intervención, informadas por la institución que asesora en gestión de riesgos a la empresa participante.

\section{Procedimiento}

Se hizo completar un consentimiento informado donde se aseguraban anonimato y confidencialidad de la investigación-acción a la organización -tanto a la gerencia como a los trabajadores-. Se realizó una evaluación diagnóstica (pre-test) sobre la base del
MCPS $^{13}$ en indicadores de accidentalidad, la satisfacción laboral ${ }^{26}$ y el compromiso organizacional. ${ }^{25}$ Posteriormente se procedió al proceso de intervención organizacional, que consistió en la ejecución de 4 etapas secuenciales y complementarias, dirigidas a impactar las 11 dimensiones del MCPS.

Primera Etapa, de Sensibilización. Buscaba impactar las dimensiones del MCPS asociadas a la actitud de la dirección, comportamiento de la dirección y participación de los trabajadores. El objetivo de esta etapa fue concientizar a todos los trabajadores de la organización (directivos, supervisores, obreros, etc.) respecto de la importancia del concepto de seguridad, tanto en el trabajo (seguridad laboral), como en otras áreas de la vida. Para ello se ejecutaron 4 acciones principales de carácter masivo consistentes en una obra de teatro (se realizaron 2 funciones para que asistiese todo el personal) que ejemplificaba la relevancia de las conductas seguras, estrategias de difusión en terreno, una jornada de facilitación con los principales actores de seguridad laboral de la organización y la intervención en los medios de comunicación de la organización para informar respecto del concepto de seguridad y del propio programa de intervención.

Segunda Etapa, de Consenso y Compromiso. Orientada a impactar las mismas dimensiones que la etapa anterior, con el objetivo de consolidarlas. En esta etapa se buscó comprometer e involucrar a todos los actores en el proceso. Para ello se construyó y difundió el protocolo de intervención, el cual fue firmado en un acto público en las dependencias de la compañía por representantes de la dirección y por los trabajadores de la empresa, así como también por los propios interventores e integrantes del equipo de investigación.

Tercera Etapa, de Implementación de la Intervención. En esta se buscó abordar todas las dimensiones del sistema de gestión de la seguridad laboral de la empresa contempladas en el MCPS.

Para la dimensión de política de prevención se llevó a cabo un taller de optimización consistente en analizar temas de planificación y política preventiva, y respuesta a emergencias. Asimismo, para la dimensión de planificación preventiva se revisó y reformuló el plan de prevención vigente en la empresa, se diseñó un catálogo de conductas seguras estandarizadas según puesto $\mathrm{y}$ tarea y se difundieron ambos a través de la realización de charlas de 5 minutos al comienzo de la jornada laboral. Por otro lado, para la dimensión de respuesta a las emergencias se llevó a cabo un taller de optimización y actualización del plan de emergencias de la empresa, donde se analizaron temas asociados a su implementación, control y difusión. Para la dimensión de comunicación se diseñó un programa de comunicación con el que se intervino diarios murales, se instalaron afiches en lugares estratégicos de la organización y se utilizó folletería para difundir información sobre el concepto de seguridad y sobre el programa propiamente tal. De igual forma, para la dimensión de formación, se diseñó un programa de capacitación en seguridad laboral, ayudando particularmente a la inducción de trabajadores que rotan en torno diferentes funciones de la empresa. En cuanto a la dimensión de control interno, se buscó potenciar los sistemas de auditoría interna en seguridad laboral de la empresa; para ello se revisó la información existente en la empresa al respecto y se plantearon mejoras desde el MCPS. Finalmente, para las dimensiones de incentivo y benchmarking se realizaron acciones tendientes a generar documentos técnicos ad-hoc a la organización. Específicamente, se analizaron las medidas de incentivo otorgadas por la empresa y se diseñó y entregó un documento 
técnico para la implementación de medidas de incentivo ad-hoc a la organización. Del mismo modo, para la dimensión de benchmarking se diseñó un documento técnico informativo sobre buenas prácticas de seguridad laboral en otras empresas del mismo sector productivo y de la misma localidad y, además, se llevó a cabo una visita guiada a una empresa de excelencia en materia de seguridad.

Cuarta Etapa de Evaluación. El objetivo de esta etapa consistió en la evaluación (pos-test) de indicadores de accidentalidad, satisfacción laboral y compromiso organizacional. Además, se realizó el cierre de la intervención informando a todos los actores involucrados los resultados de los objetivos planteados.

Todos los datos fueron procesados y analizados por medio del programa de análisis de datos estadísticos SPSS (versión 17); se utilizó análisis de frecuencias para comparar la frecuencia de accidentes reportados antes, durante y después de la intervención; además, se aplicó la prueba $\mathrm{T}$ de Student para muestras independientes para el análisis de las variables MCPS, Satisfacción Laboral y Compromiso Organizacional.

\section{RESULTADOS}

\section{Diagnóstico en Cultura Positiva, Compromiso Organizacional y Satisfacción Laboral}

Como se aprecia en la Tabla 1, en la aplicación diagnóstica (pretest) esta muestra de trabajadores mostró niveles altos de satisfacción laboral global, siendo la dimensión asociada a las prestaciones la que presentó -en promedio- niveles más bajos. Por otro lado, en cuanto al compromiso organizacional global se encontraron niveles medios y la dimensión que alcanzó menores puntuaciones fue la de compromiso continuo. Por otro lado, la cultura positiva hacia la seguridad global en la etapa diagnóstica alcanzó niveles medios, siendo la dimensión de política de prevención el componente que arrojó menores puntuaciones.

Tabla 1.

Niveles de satisfacción laboral, compromiso organizacional y cultura positiva hacia la seguridad en pre-test.

$\begin{array}{llll}\text { Dimensión } & \text { N } & \text { Media } & \text { S } \\ \quad \text { Cuestionario S10/12 } & & \\ \text { Satisfacción con las prestaciones } & 222 & 3,86 & 1,73 \\ \text { Satisfacción con el ambiente físico de la empresa } & 216 & 4,26 & 1,48 \\ \text { Satisfacción con la supervisión } & 220 & 4,38 & 1,65 \\ \text { Satisfacción Global } & 212 & 4,14 & 1,40 \\ \quad & & \\ \quad \text { Cuestionario de Compromiso Organizacional } & & & \\ \text { Compromiso afectivo } & 222 & 3,89 & 0,96 \\ \text { Compromiso normativo } & 225 & 3,65 & 1,01 \\ \text { Compromiso continuo } & 225 & 3,59 & 1,05 \\ \text { Compromiso Organizacional Global } & 218 & 3,71 & 0,82 \\ \quad \text { Escala de Cultura Positiva hacia la Seguridad } & & & \\ \text { Política de prevención } & 226 & 2,49 & 0,82 \\ \text { Incentivo a los trabajadores } & 221 & 2,79 & 0,90 \\ \text { Formación sobre riesgos laborales } & 220 & 2,85 & 0,87 \\ \text { Comunicación en materia de prevención } & 226 & 2,82 & 0,85 \\ \text { Planificación preventiva } & 226 & 2,73 & 0,96 \\ \text { Planificación de emergencia } & 227 & 3,22 & 1,01 \\ \text { Control interno } & 224 & 2,88 & 0,83 \\ \text { Técnicas de Benchmarking } & 2,97 & 0,96 \\ \text { Actitudes de la dirección hacia la gestión } & 227 & 2,71 & 0,95 \\ \text { Comportamiento de la dirección } & 225 & 2,91 & 0,90 \\ \text { Participación de los trabajadores } & 224 & 2,64 & 0,84 \\ \text { Cultura Positiva hacia la Seguridad Global } & 205 & 2,84 & 0,62 \\ & & & \end{array}$

\section{Comparación pre y pos-test en Cultura Positiva,} Compromiso Organizacional y Satisfacción Laboral

Tras no verificarse la normalidad de los datos para la mayoría de las dimensiones del MCPS, compromiso organizacional y satisfacción laboral para analizar si existen diferencias en cuanto a cada una de estas variables entre el pre y pos-test, se optó por utilizar la prueba U de Mann Whitney. La Tabla 2 muestra que existieron diferencias significativas en los puntajes globales de cultura positiva hacia la seguridad entre el antes y el después de la intervención desde el modelo, aumentando las puntuaciones en el postest. Asimismo, todas las dimensiones del MCPS mostraron mejoría significativa luego de la intervención, siendo la dimensión de técnicas de benchmarking la que mostró menor aumento.

Tabla 2.

Diferencias pre- y pos-test en Cultura Positiva hacia la Seguridad.

\begin{tabular}{|c|c|c|c|c|c|c|c|c|}
\hline Dimensión & $\mathrm{N}$ & $\begin{array}{l}\text { Pre-test } \\
\text { Mediana }\end{array}$ & Rango & $\mathrm{N}$ & $\begin{array}{l}\text { Pos-test } \\
\text { Mediana }\end{array}$ & Rango & Z & U \\
\hline $\begin{array}{l}\text { Politica de } \\
\text { prevención }\end{array}$ & 226 & 2,33 & 143,83 & 205 & 4,00 & 295,56 & $-12,69$ & $6855,0^{* *}$ \\
\hline $\begin{array}{l}\text { Incentivo a } \\
\text { los trabajadores }\end{array}$ & 221 & 2,50 & 185,16 & 206 & 3,25 & 244,94 & $-5,02$ & $16389,0^{* *}$ \\
\hline $\begin{array}{l}\text { Formación sobre } \\
\text { riesgos laborales }\end{array}$ & 220 & 2,60 & 190,33 & 200 & 3,20 & 232,69 & $-3,57$ & $17563,0^{* *}$ \\
\hline $\begin{array}{l}\text { Comunicación } \\
\text { en materia } \\
\text { de prevención }\end{array}$ & 226 & 2,66 & 179,99 & 206 & 3,33 & 256,55 & $-6,40$ & $15027,0^{* *}$ \\
\hline $\begin{array}{l}\text { Planificación } \\
\text { preventiva }\end{array}$ & 226 & 2,66 & 169,62 & 203 & 3,36 & 265,52 & $-8,03$ & $12682,0^{* *}$ \\
\hline $\begin{array}{l}\text { Planificación } \\
\text { de emergencia }\end{array}$ & 227 & 3,25 & 200,91 & 199 & 3,50 & 227,87 & $-2,26$ & $19727,5^{*}$ \\
\hline $\begin{array}{l}\text { Control } \\
\text { interno }\end{array}$ & 224 & 2,80 & 181,44 & 202 & 3,40 & 249,06 & $-5,67$ & $15441,6^{* *}$ \\
\hline $\begin{array}{l}\text { Técnicas de } \\
\text { Benchmarking }\end{array}$ & 227 & 3,00 & 205,58 & 207 & 3,00 & 230,57 & $-2,10$ & $20789,0^{*}$ \\
\hline $\begin{array}{l}\text { Actitudes de } \\
\text { la dirección } \\
\text { hacia la gestión }\end{array}$ & 225 & 2,50 & 162,25 & 203 & 3,75 & 272,41 & $-9,22$ & $11081,5^{* *}$ \\
\hline $\begin{array}{l}\text { Comportamiento } \\
\text { de la dirección }\end{array}$ & 224 & 2,80 & 180,54 & 202 & 3,40 & 250,05 & $-5,83$ & $15241,0^{* *}$ \\
\hline $\begin{array}{l}\text { Participación de } \\
\text { los trabajadores }\end{array}$ & 225 & 2,50 & 183,20 & 204 & 3,00 & 250,07 & $-5,60$ & $15796,0^{* *}$ \\
\hline $\begin{array}{l}\text { Cultura Positiva } \\
\text { hacia la } \\
\text { Seguridad Global }\end{array}$ & 205 & 2,79 & 153,43 & 182 & 3,46 & 239,70 & $-7,57$ & $10337,0^{* *}$ \\
\hline
\end{tabular}

Nota: ${ }^{* *} p<0,01 ;{ }^{*} p<0,05$.

Como se aprecia en la Tabla 3, en cuanto al compromiso organizacional los resultados indican mayores puntajes en el pos-test para el compromiso organizacional general y para dos de sus dimensiones -compromiso normativo y compromiso continuo-, en tanto que la dimensión de compromiso afectivo no presentó diferencias significativas entre el pre y pos-test.

Tabla 3.

Diferencias pre- y pos-test en Compromiso Organizacional.

\begin{tabular}{|c|c|c|c|c|c|c|c|c|}
\hline Dimensión & $\mathrm{N}$ & $\begin{array}{l}\text { Pre-test } \\
\text { Mediana }\end{array}$ & Rango & $\mathrm{N}$ & $\begin{array}{l}\text { Pos-test } \\
\text { Mediana }\end{array}$ & Rango & Z & U \\
\hline $\begin{array}{l}\text { Compromiso } \\
\text { Afectivo }\end{array}$ & 222 & 4,00 & 202,38 & 201 & 4,00 & 222,62 & $-1,70$ & 20176,0 \\
\hline $\begin{array}{l}\text { Compromiso } \\
\text { Normativo }\end{array}$ & 225 & 3,66 & 189,20 & 199 & 4,16 & 238,84 & $-4,16$ & $17146,0^{* *}$ \\
\hline $\begin{array}{l}\text { Compromiso } \\
\text { Continuo }\end{array}$ & 225 & 3,66 & 192,98 & 201 & 3,83 & 236,47 & $-3,64$ & $17995,0^{* *}$ \\
\hline $\begin{array}{l}\text { Compromiso } \\
\text { Organizaciona } \\
\text { Global }\end{array}$ & 218 & 3,77 & 181,96 & 191 & 4,00 & 231,30 & $-4,21$ & $15796,5^{* *}$ \\
\hline
\end{tabular}


Asimismo, respecto a la satisfacción laboral, se encontraron diferencias significativas entre el pre y pos-test para la satisfacción laboral general y para dos de sus dimensiones -satisfacción con las prestaciones y satisfacción con el ambiente físico de la empresa- obteniéndose mayores puntuaciones durante la segunda medición. La dimensión de satisfacción con la supervisión, por su parte, no mostró diferencias entre el antes y después de la intervención desde el MCPS (Tabla 4).

Para analizar el efecto de la intervención desde el MCPS en los niveles de accidentalidad de la empresa,en la Figura 1 se muestra la frecuencia mensual de accidentes laborales antes, durante y después de la intervención, donde se observa una reducción en la etapa post-intervención en relación a las etapas previas.

\section{DISCUSIÓN}

Esta investigación aplicada desde el $\mathrm{MCPS}^{13}$ en la empresa productiva de la zona central de Chile, dio cuenta de una disminución de la frecuencia mensual de accidentes laborales después

Tabla 4.

Diferencias pre- y pos-test en Satisfacción Laboral.

$\begin{array}{lcccccccc}\text { Dimensión } & \text { N } & \begin{array}{c}\text { Pre-test } \\ \text { Mediana }\end{array} & \text { Rango } & \text { N } & \begin{array}{c}\text { Pos-test } \\ \text { Mediana }\end{array} & \text { Rango } & \text { Z } & \text { U } \\ \begin{array}{l}\text { Satisfacción con } \\ \text { las prestaciones }\end{array} & 222 & 4,00 & 199,11 & 205 & 4,50 & 230,13 & -2,60 & 19448,5^{* *} \\ \begin{array}{l}\text { Satisfacción con } \\ \text { el ambiente fisico }\end{array} & 216 & 4,50 & 184,73 & 199 & 5,25 & 233,26 & -4,12 & 16465,5^{* *} \\ \begin{array}{l}\text { de la empresa } \\ \begin{array}{l}\text { Satisfacción con } \\ \text { la supervisión }\end{array}\end{array} 220 & 4,66 & 200,60 & 201 & 5,00 & 222,38 & -1,83 & 19823,0 \\ \begin{array}{l}\text { Satisfacción } \\ \text { Global }\end{array} 212 & 4,20 & 184,28 & 194 & 4,70 & 224,50 & -3,45 & 16489,5^{* *}\end{array}$

Nota: ${ }^{* *} p<0,01$.

Figura 1.

Distribución de la frecuencia de accidentes laborales antes, durante y después de la intervención desde el MCPS.

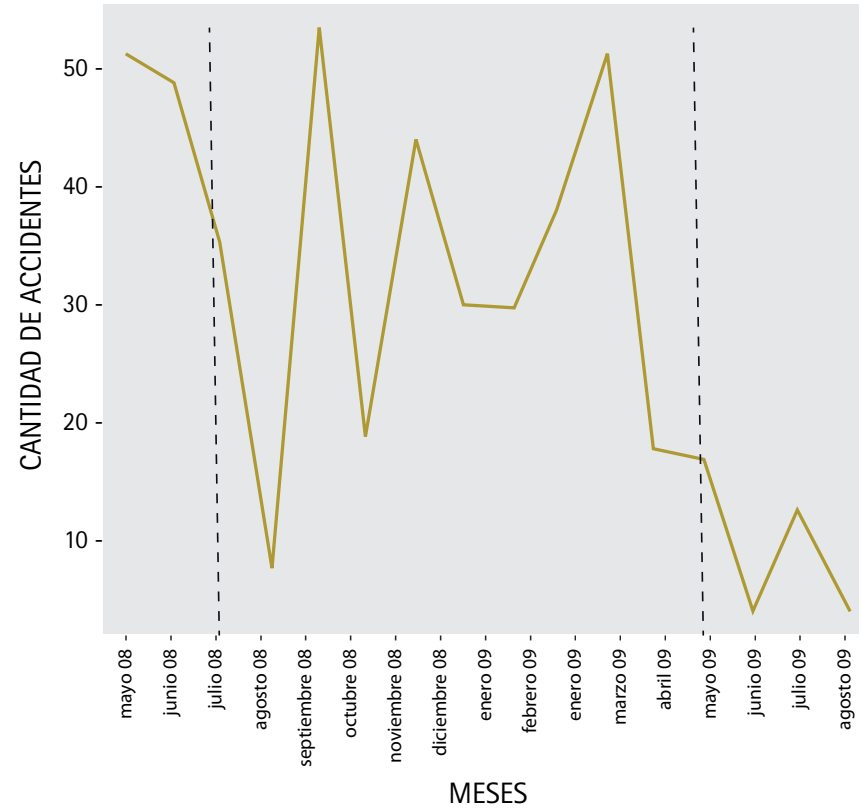

de la intervención; además, a partir del análisis pre y pos-test, se observó que tanto la puntuación global como todas las dimensiones mostraron una mejoría significativa luego de la intervención, siendo la dimensión referida a las técnicas de benchmarking la que evidenció menor aumento. En cuanto a esto, es posible que la propia naturaleza dinámica y evolutiva de las técnicas comparativas de buenas prácticas empresariales ${ }^{27}$, suponga una dificultad a la hora de establecer, adaptar y aplicar modelos externos efectivos, siendo necesarias evaluaciones constantes en un mercado de crecimiento acelerado.

En cualquier caso, la mejora de las dimensiones constituyentes del MCPS y la disminución de la frecuencia de accidentes tras la intervención dan cuenta de la importancia y necesidad de la consideración de variables asociadas al clima y cultura organizacional -y no sólo factores conductuales, discursivos o reflexivos ${ }^{12,14,15}$ - para fomentar la seguridad en ambientes laborales. ${ }^{8,13}$

Por otro lado, los análisis dieron cuenta que tanto los niveles globales de compromiso organizacional como de satisfacción laboral mejoraron significativamente luego de la intervención desde el MCPS, mostrando aumento en cada una de sus dimensiones, excepto en el compromiso afectivo y la satisfacción con la supervisión, respectivamente. Tal como plantean Allen y Meyer ${ }^{22}$, los 3 componentes del compromiso organizacional -afectivo, normativo y continuo-, se pueden desarrollar con relativa independencia, dadas sus naturalezas conceptualmente diferentes. En este sentido, el compromiso afectivo -es decir, el lazo psicológico del trabajador a través de la identificación e implicación con la organización ${ }^{25,28}$ - dependería, entre otras cosas, de características personales del trabajador y del cargo, las experiencias del trabajo y la estructura de este ${ }^{29}$, por lo que, tanto la heterogeneidad personal como organizacional (en tanto que el MCPS interviene en conjunto con todos los niveles de la organización) de la muestra podrían estar a la base de que esta dimensión del compromiso no mostrara diferencias significativas entre el pre y pos-test. Asimismo, si bien la intervención comprometió la disposición y participación de la gerencia en el establecimiento y desarrollo de la cultura positiva hacia la seguridad, el equipo interventor fue externo a la organización, y dado que los niveles basales de satisfacción ya eran altos, es posible que los empleados no hayan percibido necesariamente a la gerencia como el principal promotor de la seguridad laboral, sino que más bien hayan significado al equipo interventor como precursor del MCPS.

Existen algunas limitaciones a tener en consideración, principalmente referentes al proceso de desvinculación de un número reducido de trabajadores entre el pre y pos-test ocurrido en la empresa. Sin embargo, creemos que el MCPS, en tanto propone un abordaje del sistema organizativo como un sistema cultura ${ }^{18,13}$, puede hacer frente a modificaciones - al menos- pequeñas, no alterándose el sistema global.

De esta manera, aquí se aporta una experiencia de intervención desde la perspectiva del MCPS que permitió reducir indicadores de seguridad y aumentar niveles de satisfacción laboral y compromiso organizacional. Dado que el MCPS se encuentra en una etapa inicial de desarrollo, futuros estudios podrían abarcar otros sistemas organizacionales y sectores productivos, con otros diseños metodológicos, que permitan controlar variables que puedan estar incidiendo en los resultados para así profundizar en la evidencia de su efectividad en el ámbito de la seguridad industrial. 
1. Hämäläinen $P$, Takala J, Saarela K. Global estimates of occupationalaccidents. SafSci. 2006;44(2):137-56.

2. Hämäläinen P, Takala J, Saarela K. Global estimates of fatal work-relateddiseases. Am J IndMed. 2007;50(1):28-41.

3. Concha-Barrientos $M$, Nelson $D$, Fingerhut $M$, Driscoll $T$, Leigh J. The global burden due to occupational injury. Am J IndMed. 2005;48(6):470-81.

4. Superintendencia de Seguridad Social.SUSESO. Estadisticas de Seguridad Social 2012. Santiago: SUSESO; 2013.

5. Guzmán L. Accidentes laborales en Chile: 3 millones de días perdidos. CiencTrab. 2006;8(19):20-4.

6. Reyes M. La prevención de accidentes en el proceso enseñanza aprendizaje: una ventaja competitiva para el pais; experiencia de la Asociación Chilena de Seguridad. CiencTrab. 2006;8(19):1-5.

7. Sáez C. Gestión integrada de seguridad y salud laboral, ambiental y de calidad: una experiencia enriquecedora. CiencTrab. 2005;7(17):104-9.

8. Fernández B, Montes J, Vázquez C. Safety culture: a tool to improve corporative competitiveness. En: Ayala JC y Grupo FEDRA,editores. Conocimiento, Innovación y Emprendedores: Camino al Futuro. Logroño: Universidad de la Rioja; 2007.

9. Wiegmann D, Zhang $H$, Von Thaden $T$, Sharma G, Mitchell A. A Synthesis of Safety Culture and Safety Climate Research; Federal Aviation Administration Atlantic City International Airport, NJ. Illinois: University of Illinois; 2002.

10. Furnham A. Psicologia organizacional. El comportamiento del individuo en las organizaciones. México: Oxford UniversityPress; 2001.

11. Melià J. Un proceso de intervención para reducir los accidentes laborales. Madrid: Colegio Oficial de Psicólogos de Madrid; 1995.

12. Ugalde F. Psicologia de la conducta y seguridad industrial: Una alianza emergente. Rev Chile Psicol. 2000;21(1):69-77.

13. Fernández B, Montes J, Vázquez C. Antecedentes del Comportamiento del trabajador ante el riesgo laboral: un modelo de cultura positiva hacia la seguridad. RevPsicolTrabOrgan. 2005;21(3):207-34.

14. Komaki J, Barwick K, Scott L. A behavioral approach to occupational safety: pinpointing and reinforcing safe performance in a food manufacturing plant. J ApplPsychol. 1978;63(4):434.

15. Stave $C$, Törner $M$, Eklöf M. An intervention method for occupational safety in farming: evaluation of the effect and process. ApplErgon. 2007;38(3):35768.
16. Pearse W. Club zero: implementing OHSMS in small to medium fabricated metal product companies. J OccupHealthSafe. 2002;18(4):347-56.

17. LaMontagne $A$, Barbeau $E$, Youngstrom R, Lewiton M, Stoddard $A$, McLellan $D$, et al. Assessing and intervening on OSH programmes: effectiveness evaluation of the Wellworks-2 intervention in 15 manufacturing worksites. OccupEnvironMed. 2004;61(8):651-60.

18. Robson L, Clarke J, Cullen K, Bielecky A, Severin C, Bigelow P, et al. The effectiveness of occupational health and safety management system interventions: a systematic review. SafeSci. 2007;45(3):329-53.

19. Robbins S, Coulter M. Administración. México: Prentice-Hall Hispanoamericana; 1996.

20. Masia U, Pienaar J. Unravelling safety compliance in the mining industry: examining the role of work stress, job insecurity, satisfaction and commitment as antecedents. SAfr J IndPsychol. 2011;37(1):01-10.

21. Yusu $R$, Sari $A$. ON, The influence of occupational safety and health on performance with jobsatisfaction as intervening variables (study on the production employees). Am J Econ. 2012:136-40.

22. Allen $\mathrm{N}$, Meyer J. The measurement and antecedents of affective, continuance and normative commitment to the organization. J OccupPsychol 1990;63(1):1-18.

23. Sagie A. Employee Absenteeism, Organizational Commitment, and Job Satisfaction: Another Look. J VocatBehav. 1998;52(2):156-71.

24. Conway N, Briner R. Investigating the effect of collective organizational commitment on unit level performance and absence. J OccupOrganPsych. 2012;85(3):472-86.

25. Meyer J, Allen N. A three-component conceptualization of organizational commitment. HumResourManage R. 1991;1(1):61-89.

26. Meliá J, Peiró J. El Cuestionario de Satisfacción S10/12: Estructura factorial, fiabilidad y validez. RevPsicolTrabOrgan. 1989;4(11):179-87.

27. Yasin M. Thetheory and practice of benchmarking: then and now. Benchmarking: Intern J. 2002;9(3):217-43.

28. Mathieu J, Zajac D. A review and meta-analysis of the antecedents, correlates and consequences of organizational commitment. Psychol Bull. 1990;108:171-94.

29. Mowday R, Porter L, Steers R. Employee-Organization Linkages: The Psychology of Commitment, Absenteeism, and Turnover. New York: AcademicPress; 1982. 247 p. 